\title{
Moderne Senologie aus Sicht der Inneren Medizin
}

\section{A. Schneeweiss}

Das Mammakarzinom ist mit über 1,2 Millionen Neuerkrankungen pro Jahr die weltweit häufigste Tumorerkrankung der Frau. Trotz steigender Inzidenz sinkt deren Mortalität in vielen westlichen Industrienationen. Gründe sind neben der Einführung des Mammografiescreenings die Verbesserung der lokalen Tumorkontrolle und die effektive systemische Behandlung [1]. Adjuvante Hormon- und Chemotherapie haben das rezidivfreie Überleben und das Gesamtüberleben signifikant verbessert [2]. Neue zielgerichtet wirksame Substanzen wie Antikörper und kleine Moleküle sowie Bisphosphonate haben unsere therapeutischen Optionen stark erweitert, aber auch differenzierter und komplexer werden lassen $[3,4]$.

Parallel nimmt unser Verständnis für die molekularen und funktionellen Vorgänge bei der Entwicklung der normalen Brustdrüse und des Mammakarzinoms rasant zu. Das Mammakarzinom ist nicht länger ein einheitliches Krankheitsbild. In retrospektiven Analysen wurden wiederholt molekulare Subtypen mit unterschiedlicher Prognose und Sensitivität beschrieben, die unterschiedliche Behandlungskonzepte erfordern [5-10]. Derzeit orientiert sich die Auswahl der medikamentösen Therapien beim Mammakarzinom neben klinisch-pathologischen Faktoren nur an der Expression des Östrogen (ER)- und / oder Progesteronrezeptors (PgR) und des humanen epidermalen Wachstumsfaktorrezeptors 2 (HER2) [11,12]. Die Messung dieser Rezeptoren erfolgt in der Regel auch nur am Primärtumor, obwohl häufig metastasierte Erkrankungen behandelt werden und retrospektive Daten eine diskordante Rezeptorexpression zwischen Primärtumor und Metastase in bis zu 44\% der Mammakarzinome zeigen [13,14]. 
Es ist höchste Zeit, die One-size-fits-all-Strategie zu verlassen. Dies kann aber nur im interdisziplinären Dialog zwischen Grundlagenforschern, Diagnostikern und Therapeuten gelingen. Neue zielgerichtet wirksame Substanzen werden nur in bestimmten Subgruppen mit spezifischen Eigenschaften ausreichend wirksam sein, um eine Zulassung zu erhalten. Getriggert durch präklinisch generierte Hypothesen müssen diese Subgruppen in klinischen Studien frühzeitig anhand von Biomarkern oder funktionellen Tests definiert werden. Das neoadjuvante Therapiekonzept bildet den idealen Rahmen, um dieses Ziel zu erreichen. Es erlaubt sequenzielle Tumorbiopsien mit paralleler Bildgebung und ermöglicht dadurch eine enge Korrelation Therapie-induzierter molekularer und klinischer Veränderungen mit relevanten Endpunkten wie der pathologischen Komplettremission und damit dem Überleben. Wäre Trastuzumab ohne Vorauswahl von Patientinnen mit HER2-Überexpression getestet worden, hätte sich nie ein Überlebensvorteil gezeigt, und eines der erfolgreichsten Medikamente beim Mammakarzinom wäre wohl nie zugelassen worden. Nur durch die Konzentration auf eine definierte Subgruppe mit HER2-Überexpression konnte selbst in den frühen, kleinen Studien beim metastasierten Mammakarzinom eine signifikante und klinisch relevante Überlebensverlängerung von mehreren Monaten erzielt werden [15, 16].

Trastuzumab aber war bisher die ruhmreiche Ausnahme. Die vorherrschende Praxis ist die breite Zulassung neuer Substanzen. Dies hat zwar die Heilungs- bzw. Krankheitskontrollraten beim Mammakarzinom verbessert [2,17], aber auf Kosten einer erschreckenden Übertherapie und häufiger Fehlbehandlungen [1]. In den kommenden Jahren werden viele weitere Therapieoptionen hinzukommen [3]. Ansätze, die bis heute experimentell sind, wie zelluläre Immuntherapien, könnten eine klinische Bedeutung erlangen. Um dennoch dem Traum der personalisierten Therapie näher zu kommen, d.h. aus der rasch länger werdenden Liste an therapeutischen Möglichkeiten die richtige Therapie für die richtige Patientin zum richtigen Zeitpunkt auszuwählen, ist eine umfassende molekulare Charakterisierung des individuellen Tumors und Wirtes d.h. der betroffenen Patientin auf genomischer, epigenomischer, RNA- und Proteinebene erforderlich [18]. Moderne Hochdurchsatzverfahren erlauben uns schon heute, die molekulare Vielfalt des Mammakarzinoms und der betroffenen Patientin besser abzubilden als je zuvor. Ihre Möglichkeiten sind enorm [19]. Es gilt, sie rasch in das multimodale Behandlungskonzept des Mammakarzinoms zu integrieren. Dies kann nur durch qualitativ hochwertige Studien mit innovativen, translationalen Begleitprogrammen erreicht werden.

Vor uns liegt ein aufregender, aber auch mühsamer und langwieriger Weg mit hohen wissenschaftlichen, strukturellen und finanziellen Hürden. Lassen sie uns diese Hürden im interdisziplinären Schulterschluss gemeinsam überwinden. Wir sind es unseren Patientinnen und ihren Familien schuldig.

\section{Literatur}

1 Benson JR, Jatoi I, Keisch M et al. Early breast cancer. Lancet 2009; 373: $1463-1479$

2 Early Breast Cancer Trialists' Collaborative Group. Effects of chemotherapy and hormonal therapy for early breast cancer on recurrence and 15-year survival: An overview of the randomised trials. Lancet 2005; 365: $1687-1717$
3 Alvarez RH, Valero V, Hortobagyi GN. Emerging Targeted Therapies for Breast Cancer. J Clin Oncol 2010; 28: 3366-3379

4 Pavlakis N, Schmidt R, Stockler M. Bisphosphonates for breast cancer. Cochrane Database Syst Rev CD003474 2005

5 Perou C, Sørlie T, Eisen $M$ et al. Molecular portraits of human breast tumours. Nature 2000; 406: 747-752

6 Sørlie T, Perou CM, Tibshirani R et al. Gene expression patterns of breast carcinomas distinguish tumor subclasses with clinical implications. Proc Natl Acad Sci U S A 2001; 98: 10869-10874

7 van't Veer LJ, Dai H, van de Vijver MJ et al. Gene expression profiling predicts clinical outcome of breast cancer. Nature 2002; 415: $530-536$

8 Paik S, Shak S, Tang G et al. A multigene assay to predict recurrence of tamoxifen-treated, node-negative breast cancer. N Engl J Med 2004; 351: $2817-2826$

9 Paik S, Tang G, Shak S et al. Gene expression and benefit of chemotherapy in women with node-negative, estrogen receptor-positive breast cancer. J Clin Oncol 2006; 24: 3726-3734

10 Wirapati P, Sotiriou C, Kunkel S et al. Meta-analysis of gene-expression profiles in breast cancer: Toward a unified understanding of breast cancer sub-typing and prognosis signatures. Breast Cancer Res 2008; 10: R65

11 Goldhirsch A, Ingle JN, Gelber RD et al. Thresholds for therapies: highlights of the St Gallen International Expert Consensus on the Primary Therapy of Early Breast Cancer 2009. Ann Oncol 2009; 20: $1319-1329$

12 Beslija S, Bonneterre J, Burstein HJ et al. Third consensus on medical treatment of metastatic breast cancer. Ann Oncol 2009; 20: 1771 1785

13 Franco A, Col N, Chlebowski R. Discordance in estrogen (ER) and progestin receptor (PR) status between primary metastatic breast cancer: A meta-analysis. J Clin Oncol 2004; 22 abstr 539: 11

14 Santinelli A, Pisa E, Stramazzotti D et al. HER-2 status discrepancy between primary breast cancer and metastatic sites: Impact on target therapy. Int J Cancer 2008; 122: 999-1004

15 Slamon DJ, Leyland-Jones B, Shak $S$ et al. Use of chemotherapy plus a monoclonal antibody against HER2 for metastatic breast cancer that overexpresses HER2. N Engl J Med 2001; 344: 783 - 792

16 Marty M, Cognetti F, Maraninchi D et al. Randomized phase II trial of the efficacy and safety of trastuzumab combined with docetaxel in patients with human epidermal growth factor receptor 2-positive metastatic breast cancer administered as first-line treatment: The M77001 Study Group. J Clin Oncol 2005; 23: 4265 - 4274

17 Wilcken N, Dear R. Chemotherapy in metastatic breast cancer: A summary of all randomised trials reported 2000-2007. Eur J Cancer 2008; 44: $2218-2225$

18 Perez EA. Breast Cancer Management: Opportunities and barriers to an individualized approach. Oncologist 2011; 16: 20-22

19 Brennan DJ, Kelly C, Rexhepaj E et al. Contribution of DNA and tissue microarray technology to the identification and validation of biomarkers and personalised medicine in breast cancer. Cancer Genomics Proteomics 2007; 4: $121-134$

\section{Bibliografie}

DOI http://dx.doi.org/10.1055/s-0031-1271497

Senologie 2011; 8: 75-76

(c) Georg Thieme Verlag KG Stuttgart · New York · ISSN 1611-6453

\section{Korrespondenzadresse}

Prof. Dr. med. Andreas Schneeweiss

Sektion Gynäkologische Onkologie

Nationales Centrum für Tumorerkrankungen (NCT)

Universitäts-Klinikum

Im Neuenheimer Feld

69120 Heidelberg

andreas.schneeweiss@med.uni-heidelberg.de 